\section{Bimedial hang-back recession- outcomes and surgical response}

Manchester Royal Eye Hospital, Oxford Road, Manchester, UK

Correspondence: IC Lloyd Manchester Royal Eye Hospital, Oxford Road, Manchester M13 9WH, UK Tel: + 441612765565 .

E-mail: chris.lloyd@

cmmc.nhs.uk

Received: 17 March 2004 Accepted in revised form: 20 July 2004

Published online: 28 January 2005

\begin{abstract}
Aim To report outcomes and identify factors affecting surgical response for constant esotropia using 'hang-back' bimedial rectus recession.

Study type Retrospective case series analysis. Methods Patients managed by a single surgeon over a 4-year study period were categorized into esotropia types: infantile, partially accommodative, nonaccommodative and secondary esotropia. Postoperative alignment was compared between types, and regression modelling used to examine factors predicting surgical response.

Results In all, 95\% (18/19) of children with partially accommodative esotropia achieved postoperative deviation $<\mathbf{1 5}$ prism dioptres from orthotropia, compared to $56 \%(15 / 27)$ of children with infantile esotropia, $69 \%(11 / 16)$ of children with non-accommodative esotropia and all $(2 / 2)$ of those with secondary esotropia. Surgical response ( $\Delta / \mathrm{mm}$ recession performed) increased with the magnitude of both preoperative deviation $(P<0.001)$ and anisometropia $(P<0.001)$; the effect of deviation on surgical response was reduced by amblyopia $(P=0.02)$. Age at surgery was statistically associated $(P=0.002)$ but had negligible clinical effect on response. Conclusions Surgical response to hang-back recession may be partially predicted by preoperative factors.

Eye (2005) 19, 1178-1181. doi:10.1038/sj.eye.6701715; published online 28 January 2005
\end{abstract}

Keywords: esotropia; hang back; bimedial recession

\section{Introduction}

Successful ocular alignment following squint surgery is associated with improved cosmesis ${ }^{1}$ and psychosocial benefit. ' 'Hang-back' recession has been demonstrated to be a predictable alternative to conventional strabismus surgery, and may allow equally effective correction of ocular misalignment with lower risk of scleral perforation. ${ }^{3}$

Previous studies have identified preoperative deviation $^{4,5}$ and age at surgery ${ }^{6}$ as factors predictive of surgical response with conventional techniques. This article reports on outcomes of patients undergoing hang-back recession and examines factors predictive of surgical response with this method.

\section{Materials and methods}

Case notes from one institution (Manchester Royal Eye Hospital, UK) were reviewed of all patients undergoing 'hang-back' bimedial rectus recessions during a $4 \frac{1}{2}$ period (June 1995December 1999). All surgery and patient care was carried out or directly supervised by one surgeon (ICL). Exclusion criteria included coexisting ocular or neurological problems, previous horizontal rectus muscle surgery, or bimedial recessions combined with other muscle surgery. From the remaining 80 patients $16 \%(13 / 80)$ had insufficient follow-up; $84 \%$ $(67 / 80)$ were included in the study.

Pre- and postsurgical assessment was performed by experienced orthoptists in a hospital clinic setting. Prism cover testing was performed at $6 \mathrm{~m}$ with spectacle correction worn, as previously determined by cycloplegic retinoscopy. Prism adaptation was used to plan surgery in 12 children. Postsurgical alignment was defined as deviation at the last follow-up clinic visit, and duration of follow-up was noted in each case. Outcome was defined as cosmetically excellent if within 8 prism dioptres of orthotropia and cosmetically successful if within 15 prism dioptres of orthotropia.

Patients were categorized into esotropia types. Infantile esotropia was defined as an 
esotropia appearing before 12 months of age without a significant accommodative element or neurological deficit. Partially accommodative esotropia was defined by an increase in the deviation for near of at least 10 prism dioptres with full spectacle correction, and esotropia of $>10$ prism dioptres for distance with full spectacle correction. Esotropia not categorized above was defined as nonaccommodative when no cause for a secondary deviation was present.

Surgical response was defined as the difference between preoperative and postoperative alignment divided by millimetres of recession (rectus insertion to central muscle belly) performed. Associations between surgical response and the following preoperative factors were examined; age at onset, age at surgery, preoperative deviation for distance fixation (refraction worn), anisometropia ( $>1.25$ Dioptres), $>10 \Delta$ distance-near disparity (with refraction worn), and amblyopia ( $>3$ Snellen or equivalent line difference in acuities and vision of $<6 / 12$ in the amblyopic eye).

Statistical analysis was performed using SPSS version 11.5. Regression modelling including all factors was first performed. Nonsignificant factors $(P>0.05)$ were excluded and interactions between significant factors, residuals, leverage and influence statistics examined in a final model.

\section{Results}

Characteristics of the 67 patients are given in Table 1 . Mean age at surgery was 57 months, and mean preoperative deviation for distance fixation with corrected refraction was 35 prism dioptres. Mean duration of postoperative follow-up was 18.5 months (range 1-50 months).

Postoperatively, cosmetic excellence was achieved in $49 \%$ (33/67), and cosmetic success in $73 \%$ (49/67), of all patients. Cosmetic excellence was achieved in $26 \%(7 / 27)$ patients with infantile esotropia, $31 \%(5 / 16)$ of patients with nonaccommodative esotropia, $84 \%(16 / 19)$ of patients with partially accommodative esotropia, and in all those with secondary esotropia (2). Cosmetic success was achieved in 56\% (15/27) of patients with infantile esotropia, in $69 \%(11 / 16)$ with nonaccommodative esotropia, and in $95 \%$ (18/19) with partially accommodative esotropia. No cases of scleral perforation, A/V pattern, or surgically induced vertical deviation occurred.

Mean surgical response was $2.4 \Delta / \mathrm{mm}$ (standard deviation $1.2 \Delta / \mathrm{mm}$ ). Three patients were exotropic at postoperative follow-up (deviations of 16, 25, and $70 \Delta$ base in) and surgical responses of these cases were all outside of 3 standard deviations from mean. These outliers were subsequently excluded from analysis to avoid violation of linear regression assumptions. Age of esotropia onset, distance-near disparity $>10 \Delta$, and an accommodative component $>10 \Delta$ were not significant predictors of response. Surgical response increased with preoperative deviation $(0.69 \Delta / \mathrm{mm}$ per $10 \Delta$ esotropia, $P<0.001)$ and the presence of anisometropia $(1.1 \Delta / \mathrm{mm}$, $P<0.001)$. Age at surgery was significantly associated $(P=0.002)$ with surgical response but had a small clinical effect. Amblyopia interacted with deviation to reduce

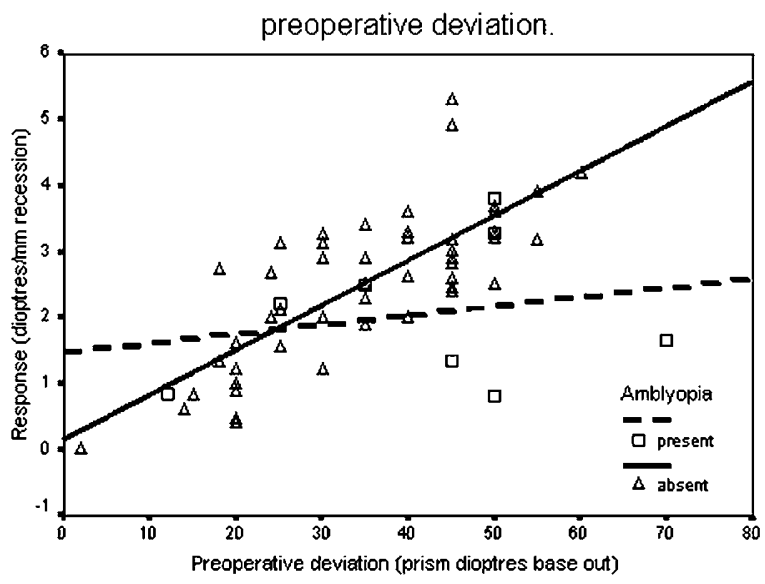

Figure 1 Surgical response by preoperative deviation

Table 1 Patient characteristics

\begin{tabular}{lcccc}
\hline Types of esotropia & Infantile & Non accomm. & Partially accomm. & Secondary esotropia \\
\hline Number & 27 & 16 & 19 & 2 \\
Age of onset (months) & 5.3 & 36 & 27 & 37 \\
Age at surgery (months) & 37 & 70 & 68 & 69 \\
Preoperative deviation $(\Delta)$ & 44 & 39 & 23 & 38 \\
Anisometropia (dioptres SE $)$ & 0.6 & 0.8 & 0.4 & 3.3 \\
Near-distance disparity $(\Delta)$ & 0.7 & 5.9 & 20 & 7.5 \\
Distance disparity with/without specs $(\Delta)$ & 1.9 & 1.9 & 2 & 0 \\
Amblyopia (frequency) & 2 & 2 & 20 & 2 \\
Length of follow-up (months) & 22 & 10 & 12 \\
\hline
\end{tabular}

${ }^{a}$ Mean value unless otherwise stated.

${ }^{\mathrm{b}}$ Spherical Equivalent. 
Table 2 Effect of preoperative factors on surgical response $(\Delta / \mathrm{mm}$ recession)

\begin{tabular}{|c|c|c|c|c|}
\hline \multirow[b]{2}{*}{ Preoperative factor } & \multicolumn{2}{|c|}{$\begin{array}{l}\text { Exploratory model }(\mathrm{n}=49 \\
\left.\quad \text { adjusted } \mathrm{R}^{2}=0.69\right)\end{array}$} & \multicolumn{2}{|c|}{$\begin{array}{l}\text { Final model }(\mathrm{n}=61 \\
\left.\text { adjusted } \mathrm{R}^{2}=0.72\right)\end{array}$} \\
\hline & $\begin{array}{l}\text { Parameter estimate } \\
\qquad(95 \% \mathrm{CI})^{\mathrm{b}}\end{array}$ & $P$-value & $\begin{array}{l}\text { Parameter estimate } \\
\quad(95 \% \mathrm{CI})\end{array}$ & $P$-value \\
\hline Mean age of onset (months) & $0.004(-0.008-0.016)$ & 0.52 & Excluded & \\
\hline Age at surgery (months) & $0.008(0.002-0.014)$ & 0.02 & $0.009(0.004-0.014)$ & 0.001 \\
\hline Pre-operative deviation ${ }^{\mathrm{a}}$ (D) & $0.07(0.05-0.09)$ & $<0.001$ & $0.07(0.05-0.08)$ & $<0.001$ \\
\hline Anisometropia $>1.25$ dioptres spherical equivalent & $1.3(0.64-1.9)$ & $<0.001$ & $1.2(0.73-1.7)$ & $<0.001$ \\
\hline Near-distance disparity $>10 \Delta$ & $0.09(-0.45-0.64)$ & 0.73 & Excluded & \\
\hline Distance disparity with/without refraction $>10 \Delta$ & $0.19(-0.33-0.71)$ & 0.46 & Excluded & \\
\hline Amblyopia & $-1.7(-2.4-1.1)$ & $<0.001$ & $-1.5(-2.0-0.93)$ & $<0.001$ \\
\hline Amblyopia $\times$ preoperative deviation ${ }^{\mathrm{a}}$ & Not applicable & & $-0.03(-0.06-0.000)$ & 0.02 \\
\hline
\end{tabular}

${ }^{a}$ Distance fixation, with refraction worn.

b95\% confidence interval.

this factor's affect on surgical response $(-0.34 \Delta / \mathrm{mm}$, $P=0.02)$; Figure 1 represents this interaction, where regression line slope is flatter for amblyopes. In all, $72 \%$ of variability in surgical response could be explained by the final regression model (adjusted $R^{2}$ value $=0.72$ ).

Of 61 cases, two outliers (with residual values 2.0 standard deviations from mean) were identified; $4.5 \mathrm{~mm}$ recessions had resulted in only $4 \Delta$ change in a $20 \Delta$ esotrope, and $7.0 \mathrm{~mm}$ recessions had produced $46 \Delta$ change in a $50 \Delta$ esotrope. Neither case had significant leverage on model parameter estimates. Associations between preoperative factors and surgical response for initial and final regression models are presented in Table 2. No multicollinearity existed between variables and assumptions underlying linear regression were valid.

\section{Discussion}

The 'hang-back' technique allows rectus recession with risk of scleral perforation theoretically lowered by needle passage through relatively thicker sclera. ${ }^{7}$ Other authors have previously compared the safety and efficacy of this technique with conventional recession surgery, ${ }^{3,8}$ but few studies have examined surgical responses with hangback surgery.

In our series, surgical response was significantly associated with various preoperative factors.

Preoperative deviation has previously been reported to correlate with response in esotropic patients. ${ }^{5}$ The same authors reported surgical response decreased with older age at surgery; paradoxically, a positive association was found in our series, although the clinical effect of surgical age was negligible. Mims et $a l^{6}$ found age at surgery caused a different dose-response plot in a series of infantile esotropes.
In the Manchester series, amblyopia interacted with preoperative deviation to lessen this factor's effect on surgical response. There are few reports examining the effect of acuity on surgical response with hang-back recession. ${ }^{5}$ Mild or moderate amblyopia has previously been reported not to influence surgical outcome with esotropia acquired after age of 1 year. ${ }^{9}$ The Manchester findings suggest, increased amounts of hang-back recession are required with amblyopia, but recognize this finding is dependent on results from only eight patients so categorized.

Anisometropia was also found to be a highly significant predictor of response. The authors suggest that anisometropia may act as a proxy for axial length; all cases with refractive asymmetry in the Manchester series were hypermetropic, and a moiety of this refractive error may presumably be due to short axial length. Kushner et $a l^{4}$ have previously

demonstrated an inverse correlation between length and response in esotropes and our findings may be attributable to increased dose responses expected with shorter eyes.

A residual deviation of less than $8 \Delta$ with the potential for microtropia has been proposed as an ideal outcome for squint surgery, leaving the patient less chance of developing a consecutive exotropia. ${ }^{1}$ Three patients in this series were exotropic post bimedial rectus recessions, although a good cosmetic outcome was subsequently achieved after a second procedure. Regression analysis including data from these patients violated linear regression assumptions, with model residuals not normally distributed. The authors' experience is that overcorrection occurring with bimedial recession surgery may later result in unpredictably large and unstable exodeviations, and suggest data exclusion allows better estimation of the recession required to achieve a cosmetically excellent result. 
A presumption of no measurement error also underlies linear regression. All data were clinically derived and not assessed for reliability. However, reproducibility was apparent through multiple pre- and postoperative measurements at successive follow-up visits, and surgical technique was standardized. The authors would suggest that the analysis findings are robust to small errors expected with intraoperative and clinical measurement of deviation, refraction, and acuity.

A proportion of the variation in surgical response in this series remains unexplained, and attributable to factors not included in our analysis. Binocularity was able to be accurately assessed preoperatively in only a limited proportion of our squint cases because of young patient ages. Prism adaptation has been used to improve accuracy of surgical outcomes and reduce frequency of overcorrection, ${ }^{10}$ but was applied in only a limited number of patients. Intraoperative variables such as surgical experience and technique, and postoperative scarring have also been suggested to affect responses. ${ }^{11,12}$ These variables are difficult to quantify, and no assessment of postsurgery scarring was attempted in this series.

While preoperative deviation, age at operation, amblyopia, and anisometropia partly predict surgical response, other factors modify immediate outcomes and maintain long-term alignment. The outcomes demonstrated within the Manchester series support the contention of Repka et $a l_{,}^{3}$ that hang-back recession method is a reasonable alternative to traditional techniques.

\section{References}

1 Willshaw HE, Keenan JM. Strabismus surgery in children: the prospects for binocular single vision. Eye 1991; 5: 338-343.

2 Mruthyunjaya P, Simon JW, Pickering JD, Lininger LL. Subjective and objective outcomes of strabismus surgery in children. J Pediatr Ophthalmol Strabismus 1996; 33(3): 167-170.

3 Repka MX, Guyton DL. Comparison of hang-back medial rectus recession with conventional recession. J Ophthalmol 1988; 95: 782-787.

4 Kushner BJ, Lucchese NJ, Morton GV. The influence of axial length on the response to strabismus surgery. Arch Ophthalmol 1989; 107: 1616-11618.

5 Abbasoglu O, Sener E, Sanac A. Factors influencing the successful outcome and response in strabismus surgery. Eye 1996; 10: 315-320.

6 Mims III JL, Treff G, Kincaid M, Schaffer B, Wood R. Quantitative surgical guidelines for bimedial recession for infantile esotropia. Binocular Vision 1985; 1: 7-22.

7 Helveston EM. Surgical management of strabismus: an atlas of strabismus surgery. Mosby.

8 Rajavi Z, Ghadim HM, Nickhoo M, Dehsarvi B. Comparison of hang-back and conventional recession surgery for horizontal strabismus. J Pediatr Ophthalmol Strabismus 2001; 38: 273-277.

9 Weakley DR, Holland DR. Effect of ongoing treatment of amblyopia on surgical treatment in esotropia. J Pediatr Ophthalmol Strabismus 1997; 34: 275-278.

10 Prism Adaptation Study Research Group. Efficacy of prism adaptation in the surgical management of acquired esotropia. Arch Ophthalmol 1990; 108: 1248-1256.

11 Lipton JR, Willshaw HE. Prospective multi centre study of the accuracy of surgery for horizontal strabismus. $\mathrm{Br} \mathrm{J}$ Ophthalmol 1995; 79: 10-11.

12 Billson FA. Accuracy in strabismus surgery. Br J Ophthalmol 1995; 79: 3. 